\title{
ASTEROSEISMOLOGY: GROUND BASED OBSERVATIONAL METHODS
}

\author{
S. FRANDSEN \\ Institute for Physics and Astronomy \\ Arhus University \\ Bygning 520 \\ DK 8000 Arhus C, Denmark
}

\begin{abstract}
The improvement in observational techniques to study pulsations in solarlike stars is discussed. For higher mass stars the problem is to allocate specific eigenmodes to a set of observed frequencies. Additional observables can be used to sort out the present ambiguities.
\end{abstract}

\section{Introduction: the photometry-astrometry connection}

The study of the interior of stars by seismic techniques imply that observations are made of periodic changes in one or more parameters. The variations looked for are as low as a few ppm (parts per million). Observers therefore strive to increase the sensitivity of the measurements as much as possible.

To reach high precision in photometry with a CCD, it is necessary to have fairly large stellar images with soft edges (Badiali et al., 1996). First, this averages out the combined effect of pointing variations and pixel calibration errors. It is impossible in practice to calibrate the response of the CCD as well as one would like, especially at the subpixel level. Thus, when the image moves around, an error signal is introduced. Secondly, sharp images will saturate the detector faster than large (defocused) images. The duty cycle decreases and precious photons are lost. Confusion with other stars puts a limit to the size of images.

To some it might be a surprise that the principles for doing precise relative photometry carry over to astrometry. The best solution for measuring precise relative positions within one CCD frame is to have large stellar images. Once more the finite size of the detector elements and related cali- 
bration problems mean that spreading the image over an area increases the accuracy.

A measuring scheme optimised for precise photometry turns out to be very close to the ideal scheme for doing precise astrometry.

If we turn to spectroscopy (one dimensional data), one can think of photometry as the measurements of equivalent widths. Astrometry corresponds to measurements of line positions, i.e. measurements of the radial velocity of the object.

Using the same arguments as in the two dimensional case, high resolution may not be the best choice for radial velocity measurements. High resolution in spectroscopy is equivalent to sharp images, and we know that this is not recommended for high precision photometry or astrometry. This is illustrated by a small diagram:

The High Precision Connections

Imaging (Two Dimensional Case)

Photometry $\Longleftrightarrow$ Astrometry

亦

Spectroscopy (One Dimensional Case)

Equivalent Width $\Longleftrightarrow$ Radial Velocity

The principles for reaching high precision should be kept in mind in the discussions that follow.

\section{Solar type oscillations: the detection}

For solar type stars with oscillations driven by stochastic excitation the main problem is to detect modes. The amplitudes are tiny for stars similar to the Sun, and even though they increase in amplitude in more luminous, hotter stars, so do the periods, and longer observing runs are needed to resolve the modes.

This section is split in three parts: first the signal to be observed is described; second, the different techniques to search for the signal are discussed and finally, the current status is given of the attempts to measure oscillations.

\subsection{WHAT DOES THE SIGNAL LOOK LIKE?}

For stars we can only make full disk measurements. Apart from a scaling of frequencies and amplitudes, we expect to find oscillation spectra similar to the solar full disk spectrum. 
The effect of the $p$ modes on the spectral distribution of light is a complicated story. The $p$ modes affect the spectral lines by the Doppler shift due to the sound velocity and by the temperature (and density) fluctuations associated with the waves. Looking at a spectral line, the intensity is affected in opposite direction on each side of the center by the velocity and in the same direction by the temperature perturbation.

The detailed behaviour of a single spectral line was studied by Andersen (1984) and Frandsen (1984). The feature to be noticed was the increase in intensity amplitude measured going towards the center of the spectral line. A much larger spectral range was measured by Ronan et al. (1991), and the results show a similar behaviour of many lines in the solar spectrum.

Looking at the results from Ronan et al. (1991) it is evident that the oscillation signal appears mainly in spectral lines. How one might combine everything into one observable (a seismic signal) is obviously not simple, especially when instrumental efficiency and atmospheric degradation of the signal have to be taken into account.

\subsection{MEASURING TECHNIQUES}

A number of different attempts have been made to try to detect oscillations in solar type stars. Doppler measurements were made first, but followed by different observations, when the required precision was obtained with Doppler measurements.

\subsubsection{Photometry}

The atmosphere is the major source of problems, when ppm level photometry is at issue. Scintillation dominates all other sources of noise for bright stars all the way down to magnitudes fainter than 10 . The noise scales with telescope diameter as $D^{-2 / 3}$. To beat down the noise by using large telescopes, a campaign was organised to try to detect oscillations in a few $\mathrm{F}$ stars in M67 (Gilliland et al., 1993).

There was no major problems in reaching the scintillation limit, so technically the campaign was a success. Unfortunately, the noise level achieved when all data were combined ( $6 \mathrm{ppm})$ was barely enough to make an unambiguous detection of modes in the $\mathrm{F}$ stars.

\subsubsection{Doppler Techniques}

The difficulty here lies in the extreme precision required that make the measurement vulnerable to instrumental instabilities of all types. The amplitude of the Doppler shift expected in a solarlike star typically corresponds to a shift of the spectrum of less than $10^{-4}$ pixel. 
The procedures applied are described in detail in the context of the search for planets around stars by Butler et al. (1996). A similar precision has been achieved with the AFOE instrument (Brown et al., 1994).

\subsubsection{Equivalent Width (EW) Techniques}

The equivalent width can be considered as an approximate indicator of the temperature. Variations are somewhat larger than flux or luminosity variations (Bedding et al., 1996). Inspired by the line of argumentation pursued in the introduction, combined with efforts to avoid the influence of the atmosphere, new observing strategies have been devised by Kjeldsen et al. (1995) and Noyes et al. (1995). Kjeldsen et al. (1995) use a type of Hydrogen line index similar to the photometric $\beta$ index. The idea is to work in a relatively narrow spectral range to reduce the influence of scintillation, and to measure something in a smooth way minimising the influence of instrumental instability and detector non-uniformities. Noyes et al. (1995) find a spectral mask by looking at the difference between spectra from two models with slightly different temperature. This looks like a very efficient method, because all the information in a wide spectral range is used. The method has to be modified to make it less sensitive to instrumental stability - the present noise levels are five times the photon noise limit. To detect oscillation in stars like $\alpha$ Cen and other solar 'twins', high efficiency is extremely important.

\subsection{OBSERVATIONAL STATUS}

A careful study of the observational status late 1993 exists (Kjeldsen \& Bedding (KB), 1995) and there is no reason to repeat what is already told. This status report thus only adds to the KB discussion.

New Doppler studies have been carried out by several groups mentioned in Table 1, which is an update of Table 4 in KB. As in Table 4 the quality of the entries should be judged by reading the references. Strictly speaking, nobody has come up with a convincing claim for a detection of modes. But in several cases the performance achieved is very close to a level, where oscillations are expected to show up either as power excess or as underlying structure in the oscillation power spectrum. Several observers find such signatures.

Photometry has come equally close, and when better measurements can be done (from space with EVRIS), it would not come as a big surprise if some of the frequencies or separation of frequencies that have been quoted are confirmed.

The equivalent width techniques have led to two claims of detection of modes, none of them confirmed by other groups. The first star with $p$ mode 
TABLE 1. Measurements to detect solar oscillations, 1994-1996

\begin{tabular}{|c|c|c|c|c|}
\hline Reference & $\begin{array}{l}v_{o s c} \\
\left(\mathrm{~cm} \mathrm{~s}^{-1}\right)\end{array}$ & $\begin{array}{l}E Q W \\
(\mathrm{ppm})\end{array}$ & $\begin{array}{l}\Delta \nu_{0} \\
(\mu \mathrm{Hz})\end{array}$ & $\begin{array}{l}\delta \nu_{0} \\
(\mu \mathrm{Hz})\end{array}$ \\
\hline \multicolumn{5}{|l|}{ - $\alpha$ Cen A } \\
\hline Edmonds \& Cram (1995) & $<70$ & - & 110.6 & - \\
\hline Kjeldsen et al. (1996) & - & 7 & $105.5 \pm 0.1$ & $6 \pm 1$ \\
\hline \multicolumn{5}{|l|}{ - $\eta$ Boo } \\
\hline Kjeldsen et al. (1995) & - & 35 & 40.3 & - \\
\hline Noyes et al. (1995) & $<150$ & & & \\
\hline Harvey et al. (1996) & \multicolumn{4}{|c|}{ test observations } \\
\hline \multicolumn{5}{|l|}{ - Procyon } \\
\hline Bedford et al. (1995) & $<100$ & - & $70 \pm 4$ & - \\
\hline Brown et al. (1996) & \multirow{2}{*}{\multicolumn{4}{|c|}{$\begin{array}{l}\text { no data published } \\
\text { test observations }\end{array}$}} \\
\hline Harvey et al. (1996) & & & & \\
\hline Kjeldsen et al. (1996) & - & $20-25$ & 52.6 & - \\
\hline Noyes et al. (1996) & \multicolumn{4}{|c|}{ test observations } \\
\hline \multicolumn{5}{|l|}{ - $\beta$ Hyi } \\
\hline Edmonds \& Cram (1995) & $<150-200$ & - & - & - \\
\hline \\
\hline
\end{tabular}

data was $\eta$ Boo (Kjeldsen et al., 1995) and the second one $\alpha$ Cen (Kjeldsen et al., 1996).

To summarise I will give the status for four different objects. Other stars have been observed, but the main efforts have been devoted to these targets.

1. The Sun. The Sun has served as a reference object for testing the equivalent width techniques. There has been discussion about whether any signal appeared in the Hydrogen lines at all. The measurements by Ronan et al. (1991) indicated that the Balmer lines vary only weakly. A signal with an amplitude of the order $6 \mathrm{ppm}$ (as predicted) was seen in daytime observations of the blue sky, both in the case of the $\eta$ Boo observations and the $\alpha$ Cen observations. The latest solar data obtained during the $\alpha$ Cen campaign gives the large separation of 135 $\mu \mathrm{Hz}$ within a tenth of a $\mu \mathrm{Hz}$. Harvey et al. (1996) observed the $\mathrm{H} \alpha$ line in the Sun at Kitt Peak and were able to confirm the presence of a signal with expected amplitude. Thus, there seems to be no doubt, that the signal shows up in the Balmer lines at the expected amplitude. 


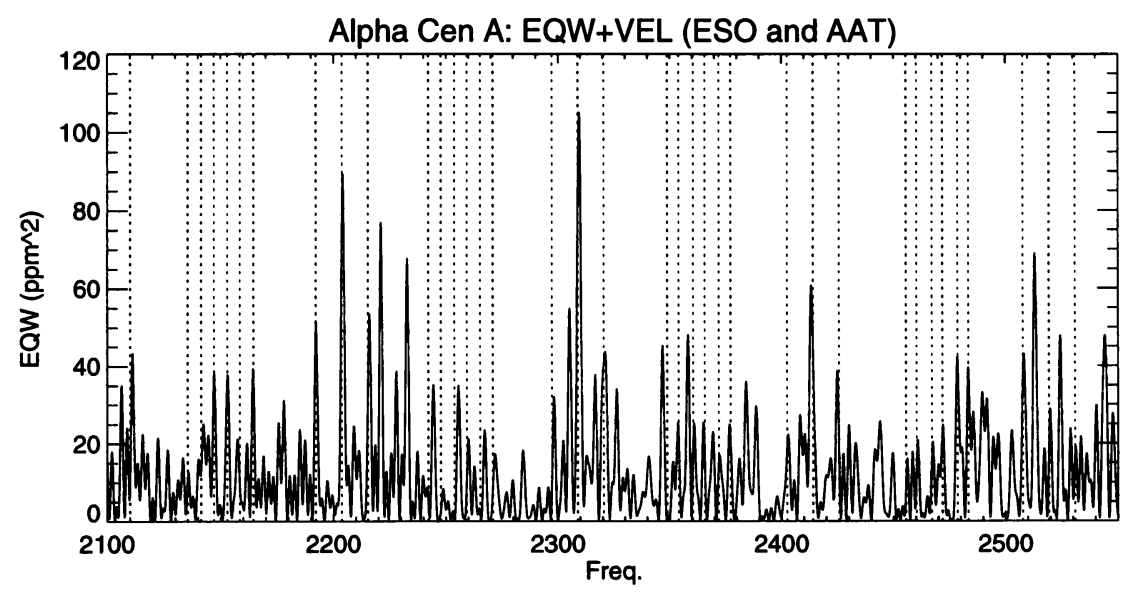

Figure 1. The power spectrum from the $\mathrm{H} \alpha$ line measurements combining an equivalent width and a velocity. The dashed line indicates the expected position of peaks with the large and small separation that have been selected (see text)

2. Procyon. Nobody has been able to observe single modes, but there seems to be excess power in the right frequency band around $1.5 \mathrm{mHz}$ (see references in KB). New observations confirm the excess (Kjeldsen et al., 1996). One can still discuss the significance of the data (as KB do), and a new, large observing run is needed to find out, whether the power seen is instrumental or not. A campaign is planned early 1997 under the auspices of the SONG (see later) initiative.

3. $\eta$ Boo. The detection of oscillations in this star by Kjeldsen et al. (1995) still awaits confirmation. An attempt by Noyes et al. (1995) to reobserve the modes did not show any of the formerly detected modes. The discrepancy is still being disputed.

4. $\alpha$ Cen A. This binary component is a key object for stellar modelling and seismology, due to its similarity to the Sun. A large operation involving the ESO $3.6 \mathrm{~m}$ and the AAT $3.9 \mathrm{~m}$ telescopes for 6 consecutive nights was launched to try to detect modes with EW amplitudes expected at a level of $7 \mathrm{ppm}$ (Kjeldsen et al., 1996). There is evidently a tendency for peaks to show up at equidistant intervals in the power spectrum (Fig. 1). The peaks are mostly in the range 2-4 times the noise, and none of them are unambiguous detections in their own right. This regularity of peaks around $2.3 \mathrm{mHz}$ permits a determination of the large separation $\Delta \nu=105.48 \pm 0.1 \mu \mathrm{Hz}$. The small separation has been determined as well, $\delta \nu=6 \pm 1 \mu \mathrm{Hz}$. The combination of this value and the two site alias peaks leads to some problems in selecting the correct large separation. 


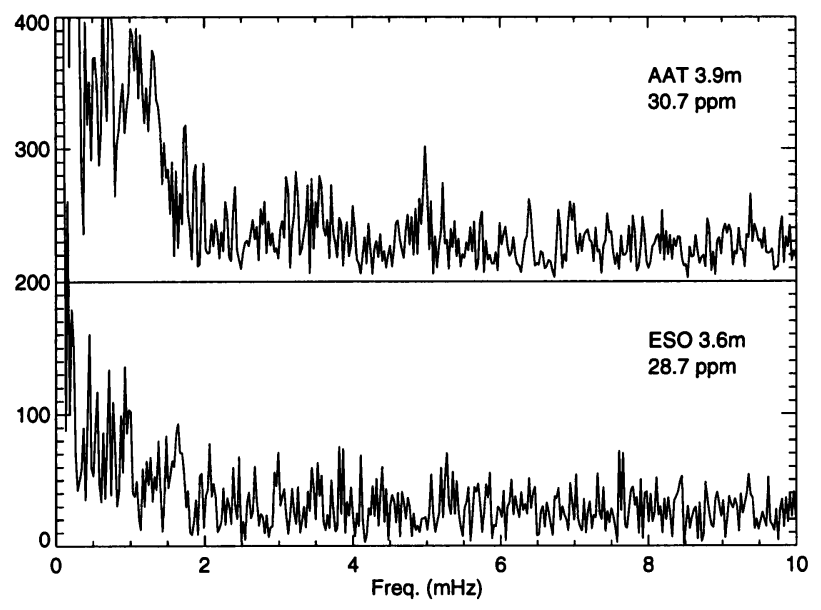

Figure 2. The amplitude spectrum of a continuum index formed by comparing two narrow $(0.5 \AA)$ bands $1 \AA$ apart. The spectra hae been generated from 10 hours of observations at each site. The photon noise is $10-12 \mathrm{ppm}$. The power is seen to be larger at the AAT site than at ESO and to have a stronger $1 / \mathrm{f}$ component. Vertical axis is in ppm. White noise levels are given for each site. The difference in noise levels corresponds to the difference in airmass at the two sites.

The photon noise limit is not reached for reasons that begin to be understood. One of the limitations of the equivalent width measurements is the differential effect of scintillation. Even for a small piece of the spectrum the continuum is seen to wobble like a rubber band. A comparison of an index of the variation in the continuum shows a stronger variation at the Australian site, which is situated at lower altitudes than La Silla and more sensitive to atmospheric conditions. This can be seen in Fig. 2.

This has as a consequence, that the ESO data turn out slightly better, even though a more stable spectrograph was used on a larger telescope at the AAT .

Recent years have moved the observational limit to the ppm levels if large telescopes are used. It is not a question whether we are able to detect solar type oscillations unambiguously. The question is how soon? Large telescopes are not necessary, if the photons are used in an efficient way. The latest $\alpha$ Cen results prove that we can reach noise levels that allow us to measure oscillations in stars like Procyon and $\eta$ Boo.

It is also clear that, when using the Balmer lines to look for oscillations at equivalent width amplitudes below $10 \mathrm{ppm}$, the atmosphere has a disturbing influence. There are still many experiments to be performed before an optimal technique has been developed, which circumvent the detrimental 
effects of the atmosphere and instrumental stability and still makes good use of the photons.

A working group: Stellar Oscillations Network Group (SONG) has been formed to pursue observations and technical development in this direction.

Recently, a special class of variables have been identified by Edmonds and Gilliland (1996). They find variability in $\mathrm{K}$ giants with large amplitudes (5-15 mmag) and long periods (1-2 days), which might be $p$ modes. They would represent an extension to giant stars of the stochastically excited oscillations. The amplitudes are high enough that they can be observed from the ground, but very long observing runs are needed to give a frequency resolution high enough to see individual $p$ modes.

\section{The 'classical' variables: identification of modes}

These variables generally have amplitudes that are easily measured, even with one channel photometers on a few nights. When more efficient and extensive observing programmes are invoked, many more low amplitude modes appear. A multitude of modes makes asteroseismology much more interesting, and the efforts involved in using more advanced measurements are fully compensated by the wealth of information one recovers.

I will mainly discuss $\delta$ Scuti stars, but much of the discussion and the results apply to $\beta$ Cephei, roAP stars and other short period $(P<4 \mathrm{~h})$ variables.

\subsection{WHAT DO WE NEED TO CLASSIFY?}

The $\delta$ Scuti stars often rotate with a considerable velocity, typically $V \sin i=$ $100 \mathrm{kms}^{-1}$. Evolved stars have complex oscillation frequency spectra with avoided crossings and modes that are a mix of $g$ and $p$ modes. Even when a large set of modes (>20) have been observed (Breger et al., 1995), these have to be matched to a set of theoretical modes, which is 3-4 times larger. If only the observed frequencies are known, it is not an easy task.

If we could determine, not only the frequency, but also the character of the mode in terms of the angular quantum numbers $\ell$ and $m$, the match of observed and theoretical frequencies would be much easier.

A number of techniques have been suggested to determine $\ell$ and possibly $m$, which I will discuss one by one. I will also present a few examples of results obtained recently.

\subsection{PHOTOMETRY}

Observations of the variables must resolve the oscillation spectra. Modes are typically found with separations down to one or two $\mu \mathrm{Hz}$ or less. New 
observations confirm what has been expected for a long time: more modes appear when the detection level decreases. Observations from one site consequently must have an extraordinary $\mathrm{S} / \mathrm{N}$ to make it possible to handle the alias problems created by the daily side lobes. The photometric data needed for asteroseismic studies can in practice only be obtained by extensive multisite campaigns. Excellent results have been obtained by the DSN, STEPHI and the WET groups, the best results when the two networks (DSN+WET) work together (Breger et al., 1995).

As more modes show up every time the detection level is lowered, efficient measuring techniques are important. In comparison to photoelectric photometry, relative CCD photometry gives an important increase in precision per minute of observation time. It comes at the cost of more complicated operations and more data reduction. It depends on the availability of reference stars within the field of view, but it is less of a problem now than a few years ago with the advent of large format detectors.

There is a big gain in efficiency, when many stars can be observed within a single CCD frame. A few open clusters that permit simultaneous measurements are known, but with a small set of variables (2-6). Praesepe (Belmonte et al., 1996) has a rich population of $\delta$ Scuti stars (> 10 multiperiodic pulsators), but at most telescopes at most two stars can be observed simultaneously. Needless to say, the membership of an open cluster provide you with some very useful additional information, when modelling the evolution of the cluster.

\subsection{MODE CLASSIFICATION}

The techniques for identifying the quantum numbers characterising a mode depends on the effect on the observable of the spherical function belonging to an eigenmode. The difference in the center to limb behaviour of the observable combined with the usual limb darkening gives a different amplitude and phase for each spherical wave function. Some degeneracy is present when the star is a slow rotator. For moderate to fast rotators the line profiles can be analysed using Doppler imaging techniques. This works well for moderate to high degree modes $(\ell \gg 1,|m| \approx \ell)$.

Observations of phase differences between colours and magnitudes were among the first methods applied. Recent extensions of the method to include amplitude ratios is the reason for having the discussion of this technique at the end of the section.

\subsubsection{Moment Methods}

The influence of oscillations on the spectral line profiles was studied early by Balona (1986a), and a mode identification technique developed in a series 
of papers $(1986 \mathrm{~b} ; 1987)$. Later improvements are due to Aerts $(1992 ; 1996)$. Recent examples of the use of the moment method are Mathias \& Aerts (1996) and Mantegazza \& Poretti (1996).

The advantages of the technique is that the moments are easy to calculate directly from the observed profiles. The moments describe a decreasing series in spatial scale size on the stellar surface. The theoretical interpretation is not straight forward. It involves the computation of the change of spectral line profiles caused by the oscillations, and the subsequent generation of the moments for combinations of the independent parameters in the problem. Not only the quantum numbers enter, but also the rotation velocity, the inclination of the rotation axis and a few more.

Often the result is a small selection of possible solutions that all fit equally well.

The method demands that the star does not rotate fast. In fast rotating stars most lines overlap and the simple picture of one line containing the velocity information disappears. If only applied to one or a few lines, it is not a method with a very high $\mathrm{S} / \mathrm{N}$ as very few photons are involved.

\subsubsection{Doppler Imaging}

The broadening effect of the rotation and the oscillations on the spectrum can be derived using deconvolution techniques. By taking out the mean effect caused by rotation one is left with the combined effect of all modes. Given a time series of spectra one then generate a two dimensional Fourier diagram with time information in one direction and spatial information in the other. A beautiful example is presented for $\tau$ Peg at this meeting by Kennelly et al. (1996), where the final 2D diagram contains so many frequencies that it reminds one about a helioseismic $k-\omega$ diagram..

The power of the technique lies in the ability to use the whole spectrum. Blends of lines can be included without difficulty. A decent initial estimate of the spectrum is needed and can be obtained from a synthetic spectrum of a model atmosphere with effective temperature close to the temperature of the star being analysed.

Doppler imaging works well for high $\ell$ values, but it looses its sensitivity at low $\ell$ values. It is the only method that can detect high $\ell$ oscillations. So far, there is a gap between the low and high $\ell$ values, where none of the methods work well. This makes it difficult to relate frequencies for low degree photometric frequencies to high degree Doppler imaged oscillation frequencies.

An assumption being made in the Doppler imaging approach is that all lines react in the same way. Some difference can be accepted because the average behaviour is normally what one is looking for. But, in an example mentioned below, various metal lines do not react in the same way. For 
$\delta$ Scuti stars the assumption is quite reliable, as the modes are well below the acoustical cutoff frequency.

\subsubsection{Amplitude Ratio and Phase Difference Techniques}

The use of phase shifts in multicolour photometry to identify modes has been suggested long ago. Even better diagnostics can be found by including more observables. The radial velocity decreases more toward the limb than the flux measured in various passbands. The same is true for the equivalent width of the Balmer lines. Each observable defines a spatial filter. Combining these filters with the spherical functions give different amplitudes for different $\ell$ (and $m$ ) values. When taking the ratios of the amplitudes for different observables the intrinsic amplitude of a mode cancels out as well as the phase. This ratio and phase difference then characterise the mode and can be used to label the mode. Some ratios are more sensitive than others and give a better discrimination between different $\ell$ values. Amplitude ratio and phase results for $\delta$ Scuti stars observed in the Strömgren system are given by Garrido et al. (1990). Calculations for $\beta$ Cephei stars have been carried out by Cugier et al. (1994). They show the separation of modes with different $\ell$ 's in a diagram of $K / A_{y}$ vs. $A_{u-y} / A_{y}$, where $\mathrm{K}$ is the radial velocity amplitude and $A_{y}$ and $A_{u-y}$ the amplitudes of the magnitude and colour in the Strömgren system.

For a star with a spectrum of detected frequencies, one can derive the amplitude ratios for given frequencies. Then a good window function is not a necessity for obtaining precise ratios.

Some observables can be measured with great precision. Velocities can be obtained from many lines in several orders of the spectrum, and only a moderate resolution is adequate $(\Delta \lambda=0.5 \AA)$, as the rotational broadening is in general quite large. The strength of one or more lines (Balmer or metal) is another observable that can be measured in an efficient and accurate way.

Observations of this type are shown by Viskum et al. (1996) for the $\delta$ Scuti star FG Vir. The power spectrum of a time series in the Doppler velocity is presented as Fig. 3. All frequencies detected by Breger et al. (1995) are present. The data is only partially reduced and no information on mode identification is available yet.

Another exciting achievement, by Baldry et al. (1996) using Doppler studies, is the measurement of the velocity spectrum of the roAp star $\alpha$ Cir, where most of the peaks seen in photometric measurements (Kurtz et al., 1994) are present. The lowest amplitude detected so far is around $30 \mathrm{~ms}^{-1}$. A very interesting observation, made during the reduction, was that the metal lines fell in two groups with opposite phase. The hypothesis is that the eigenfunction has a node in the atmosphere as the star is not heavily spotted. This has some interesting consequences. In the Sun the $p$ modes are 


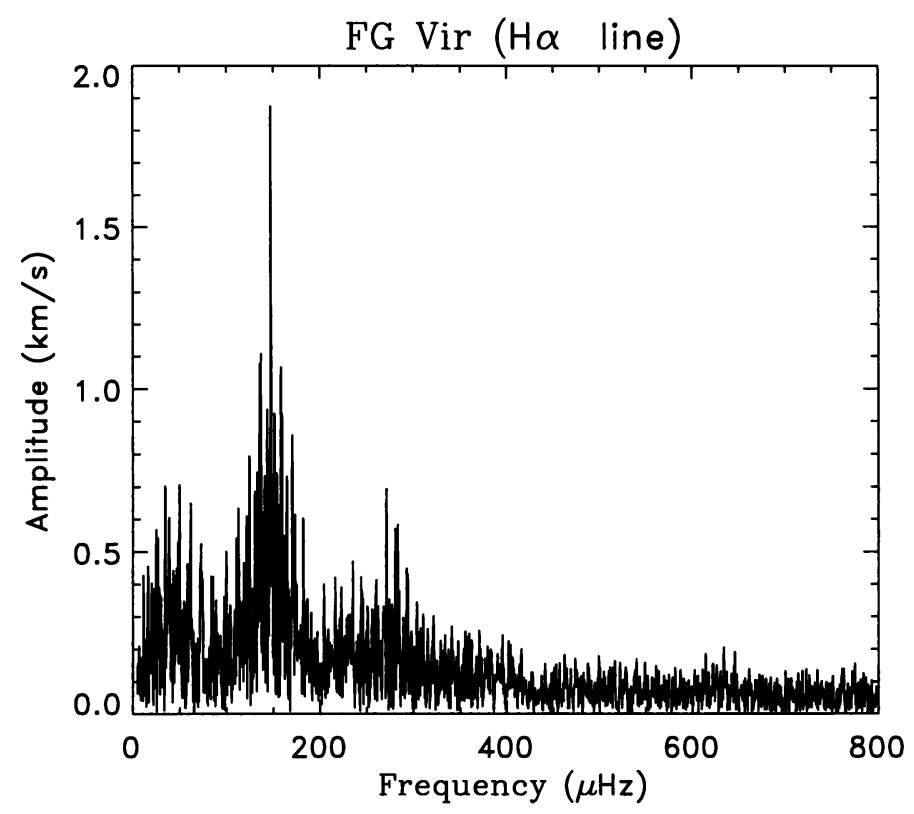

Figure 3. An amplitude spectrum of Doppler measurements of FG Vir. The white noise level above $400 \mu \mathrm{Hz}$ is $64 \mathrm{~ms}^{-1}$. Data from (Viskum et al., 1996).

evanescent in the atmosphere and no nodes can be found. In the roAp star the reflecting boundary has to be situated very far out, if the interpretation of the data is correct. It is consistent with the fact that the modes are high overtones with a frequency very close to the acoustical cutoff frequency.

While discussing spectral techniques and the sensitivity of observables, it might be worthwhile to discuss the best technique to reach low noise levels. CCD photometry works fine for amplitudes down to $50-100 \mathrm{ppm}$. A hard limit is reached, when scintillation noise is the dominating source of noise. In this situation it turns out, that one can do better in the same amount of time by using an observable, which is insensitive to scintillation. For bright stars, using velocity measurements, one might easily find that the $\mathrm{S} / \mathrm{N}$ per time unit is smaller than by applying photometry. Even fainter modes can be detected than with CCD photometry.

A final word concerns the range of modes detectable by full disk measurements in slow as well as fast rotating stars. The velocity signal has a spatial filter function that still lets an $\ell=3$ mode pass without too much attenuation. In equivalent width measurements indications of the $\ell=3$ mode have been seen in the Sun (Bedding et al., 1996). Observables probably exist that extend the range to even higher $\ell$ 's. Doppler imaging, of course, goes even higher, but is limited to fast rotators. 


\section{The Future}

Asteroseismology, based on observations of a rich set of modes, is now just around the corner. Data for solar type stars have been presented, but need confirmation.

Rich set of modes exist for a few $\delta$ Scuti stars and I expect soon to see additional data show up. The modelling of the stars and the inversion of these data should soon be possible giving a lot of new information about the interior of stars with masses in the range 1.5 to $2 M_{\odot}$.

Considering the interest in setting up networks of instruments (DNS, STEPHI, SONG) to observe solar type stars as well as higher amplitude variables, an analysis of the best choice of instrument to use is of extreme importance. A lot of money and manpower is involved and a bit of thought on how to get most out of the investments is recommended.

The attempts of the detection of oscillations in solar type stars have led to lots of ideas about the most precise and efficient techniques. Observers of the classical variables should keep in mind that the same ideas apply when deciding about instrumentation for observing $\delta$ Scuti stars etc. White dwarfs are an exception due to the lack of spectral lines.

For bright stars, my personal opinion is that a simple spectrograph of intermediate dispersion, covering a wide spectral range in several orders, is the best bargain for a network instrument. The optimum solution might be to have both a multichannel photometer and a spectrograph, but if one has to choose one or the other, I believe a spectrograph is the prime instrument of the two. It is also the most costly, to build and to operate.

\section{References}

Aerts, C., De Pauw, M. and Waelkens, C. (1992) A\&A 266, 294

Aerts, C. (1996) A\&A 314, 115

Andersen, B.N. (1984) in Proc. of the 25th Liège Int. Astr. Coll., 220

Badiali, M., Catala, C., Favata, F., Fridlund, M., Frandsen, S., Gough, D.O., Hoyng, P., Pace, O., Roca-Cortés, T., Roxburgh, I.W., Sterken, C. and Volonté, S. (1996) STARS, Seismic Telescope for Astrophysical Research from Space, Report on the phase A Study, ESA report SCI (96) 4

Baldry, I.K., Viskum, M., Kjeldsen, H., Frandsen, S. and Bedding, T.R. (1996) This meeting, the poster proceedings

Balona, L.A. (1986) MNRAS 219, 111

Balona, L.A. (1986) MNRAS 220, 647

Balona, L.A. (1987) MNRAS 224, 41

Bedding, T.R., Kjeldsen, H., Reetz, J. and Barbuy, R. (1996) MNRAS 280, 1155

Bedford, D.K., Chaplin, W.J., Coates, D.W., Davies, A.R., Innis, J.L., Isaak, G.R. and Speake, C.C (1995) MNRAS 273, 367

Belmonte, J.A., Hernández, M.M., Michel, E., Álvarez, M., Jiang, S.Y. and the STEPHI network (1996) This meeting, the poster proceedings

Breger, M., Handler, G., Nather, R.E., Winget, D.E., Kleinman, S.J., Sullivan, D.J., Li, Z.-P., Solheim, J.E., Jiang, S.-Y., Liu, Z.-L., Wood, M.A., Watson, T.K., Dziem- 
bowski,W.A., Serkowitch, E., Mendelsohn, H., Clemens, J.C., Krzesinski, J. and Pajdosz, G. (1995) A\&A 297, 473

Brown, T.M., Noyes, R.W., Nisenson, P., Korzennik, S.G. and Horner, S. (1994) PASP 106, 1285

Brown, T.M., Kennelly, E.J., Noyes, R.W., Korzennik, S.G., Nisenson, P., Horner, S.D. and Catala, C. (1996) BAAS 188, 5902B

Butler, R.P., Marcy, G.W., Williams, E., McCarthy, C. and Dosanjh, P. (1996) PASP 108,500

Cugier, H., Dziembowski, W.A. and Pamyatnykh, A.A. (1994) A\&A 291, 143

Edmonds, P.D. and Cram, L.E. (1995) MNRAS 276, 1295

Edmonds, P.D. and Gilliland, R.L. (1996) ApJ 464, L157

Frandsen, S. (1984) in Proc. of the 25th Liège Int. Astr. Coll., 303

Garrido, R., Garcia-Lobo, E. and Rodríguez, E. (1990) A\&A 234, 262

Gilliland, R.L., Brown, T.M., Kjeldsen, H., McCarthy, J.K., Peri, M.L., Belmonte, J.A., Vidal, I., Cram, L.E., Frandsen, S., Parthasathy, M., Petro, L., Schneider, H., Stetson, P.B. and Weiss, W.W. (1993) AJ 106, 2441

Harvey, J., Pilachowski, C., Barden, S., Giampapa, M., Keller, C. and Hill, F. (1996) BAAS 188, \#59.03

Kennelly, E.J., Brown, T.M., Sigut, A., Noyes, R.W., Korzennik, S.G., Nisenson, P., Horner, S.D., Yang, S. and Walker, A. (1996) This meeting, the poster proceedings

Kjeldsen, H., Bedding, T., Viskum, M. and Frandsen, S. (1994) AJ 109, 1313

Kjeldsen, H. and Bedding, T.R. (1995) A\&A 293, 87

Kjeldsen, H., Frandsen, S., Bedding, T., Dall, T. and Christensen-Dalsgaard, J. (1996) in SONGNews 1, http://www.noao.edu/song/

Kurtz, D.W., Sullivan, D.J., Martinez, P. and Tripe, P. (1994) MNRAS 270, 674

Mantegazza, L. and Poretti, E. (1996) A\&A 312, 855

Mathias, P. and Aerts, C. (1996) A\&A 312, 905

Noyes, R.W., Korzennik, S.G., Nisenson, P., Brown, T.M., Kennelly, T. and Horner, S. (1995) BAAS 187, \#102.11

Noyes, R.W., Korzennik, S.G., Krockenberger, M., Nisenson, P., Brown, T.M., Kennelly, T. and Horner, S. (1996) BAAS 188, \#59.06

Ronan, R.S., Harvey, J.W. and Duval, T.L. (1991) ApJ 369, 549

Viskum, M., Baldry, I.K., Kjeldsen, H., Frandsen, S. and Bedding, T.R. (1996) This meeting, the poster proceedings 